\title{
El legado profesional como patrimonio institucional \\ La obra bibliotecológica de Hanny Stoecker de Simons en la Biblioteca Pública de la Universidad Nacional de La Plata
}

\author{
( Julián Meclazcke \\ Universidad Nacional de La Plata. Facultad de Humanidades y Ciencias de la Educación I \\ julianmeclazcke@gmail.com / https://orcid.org/oooo-0003-0884-6919
}

\begin{abstract}
Resumen
Hanny Stoecker de Simons fue vicedirectora de la Biblioteca Pública de la Universidad Nacional de La Plata durante tres décadas (1919-1949) en las que esta institución fue refundada en todos sus aspectos, servicios y significaciones. Desde la arquitectura hasta la organización de los estantes, su huella se constituyó como incógnita, perdiéndose en la pompa de las declaraciones oficiales. En el presente trabajo se plantea su figura como objeto de estudio, entendiendo que toma el carácter de demanda y necesidad histórica. Su condición de mujer bibliotecaria es analizada desde una perspectiva de género, con una metodología autóctona de la disciplina, al tiempo que su génesis profesional se desarrolla siguiendo los parámetros de los estudios biográficos afines. Se espera poder visibilizar el impacto de Simons en el desarrollo de la Biblioteca Pública de la UNLP, analizando sus aportes a la organización del fondo documental y la reglamentación de los préstamos domiciliarios. Se recorre además su obra intelectual, con el objetivo de ponderar su contribución al desarrollo del campo bibliotecológico local.
\end{abstract}

Professional legacy as institutional heritage: the librarianship work of Hanny Stoecker de Simons in the Biblioteca Pública of the Universidad Nacional de La Plata

\footnotetext{
Abstract

Hanny Stoecker de Simons was deputy director of the Biblioteca Pública of the Universidad Nacional de La Plata for three decades (1919-1949) in which this institution was re-founded in all its aspects, services and meanings. From the architecture to the organization of the shelves, her track fades behind in the pomp of official declarations. Her figure is considered here as an object of study, understanding it as an historical demand and necessity. Her status as a female librarian is analyzed from a gender perspective, with a native methodology of the discipline, while her professional development is followed parameters of related biographical studies. It is expected to be able to visualize the impact of Simons in the development of the UNLP Public Library, analyzing her contributions to the organization of the library collection and Esta obra está bajo una Licencia Creative Commons Atribución-Compartirlgual 4.0 Internacional.
}

\section{Palabras clave}

Biblioteca Pública Universidad Nacional de La Plata Estudios feministas Bibliotecología Historia de las bibliotecas Inmigración científica Argentina

\section{Keywords}

Biblioteca Pública Universidad Nacional de La Plata Feminist studies Library Science Libraries History Scientific Immigration Argentina 
the regulation of home loans. Her intellectual work is also explored, with the aim of weighing her contribution to the development of the local library science field.

Artículo recibido: 16-02-2020 Aceptado: 10-05-2020

\section{Introducción}

En las primeras décadas del siglo veinte, la bibliotecología argentina daba los pasos iniciales hacia la profesionalización del campo. Entre hitos y figuras fundacionales, el campo de estudios fue nutrido por aportes teóricos y empíricos de distinto orden que le dieron sus primeros lineamientos. Sin embargo, dadas las características y los cánones imperantes en este período de nuestra historia y de la historia latinoamericana en general, las contribuciones profesionales llevadas a cabo por mujeres raramente llegaron hasta nuestros días por la vía escrita.

Como parte estructural del sistema educativo-cultural, las bibliotecas constituyeron espacios en los cuales la inserción femenina fue objeto de debates desde los primeros momentos de su existencia. Según Planas (2017) la mujer pudo acceder por primera vez a estos espacios favorecida por la presidencia de Sarmiento y, en nuestro campo, por las políticas de la Comisión Protectora de las Bibliotecas Populares. Esta propiciaba, no sin algunas contradicciones, la creación de un perfil lector en la mujer y su participación en la gestión bibliotecaria. Los cambios que acarrearía más tarde la figura del inmigrante, el respectivo brote de choques culturales e ideológicos y la ampliación gradual y no lineal del acceso a la lectura no tardarían en obtener una respuesta del orden conservador, con la aparición de las políticas profilácticas y moralistas que pondrían un cerco a la elevación cultural de la mujer, aduciendo toda clase de peligros, que variaban desde la desviación psicológica hasta la sexual, entre otros puntos extremos.

Al ritmo que eran superadas las restricciones culturales y sociales que había impuesto el régimen conservador, las bibliotecas aún se entendían como una extensión del espacio doméstico; si bien libre de la disciplina escolar, se esperaba que la mujer propiciara un clima similar al de la casa. Representaban además mano de obra barata y su función se asemejaba en algunos aspectos al rol docente, vinculado siempre a la maternidad. A la vez se esperaba que ejecute esas tareas reiterativas que, según Dewey (Rodríguez Toajas, 2013), no se diferenciaban y eran adecuadas para ellas “... por su atención a los detalles, su salud endeble y su incapacidad para la administración". La sociedad norteamericana fue pionera en esta inserción, a fines del siglo diecinueve. Esta visión no tardó en llegar a nuestro país, en primer lugar importada para implementarse en todo el espectro cultural por Sarmiento; décadas más tarde sería desde el mismo campo, debido al seguimiento que se realizaba a las innovaciones norteamericanas. Hacia la década de los años veinte las mujeres ya trabajaban en las bibliotecas, y podían disponer de suficiente responsabilidad como para reconfigurar sus instituciones. Siendo aún consideradas inferiores en los ámbitos de dominio masculino, y siendo ellas mismas víctimas de este discurso hegemónico que las cohibía de la participación en los desarrollos "más elevados", en donde sí podemos apreciar su capacidad es en la obra profesional; en los cambios que propiciaron en sus bibliotecas y, dado el caso, en su obra intelectual (Gutiérrez y Romero, 1989).

1. En adelante Hanny Simons, como era su signatura personal.
Hanny Stoecker de Simons ${ }^{1}$ fue vicedirectora por casi tres décadas de la Biblioteca Pública de la Universidad Nacional de La Plata (UNLP). Su nombre aparece en la bibliografía como una confidencia de autores que consideran necesario decirlo, 
recordarlo, confirmarlo al paso, una costumbre o tradición que se repite desde Angélica Costa Álvarez de Sapin, su colega, hasta Amelia Aguado de Costa, su heredera. Transmiten la información en fragmentos, como a la espera de una eventual reunión, largamente postergada, de índole compleja y con el peligro del olvido siempre latente y amenazante. La ligan a las reformas que presentan los primeros directores de la institución universitaria. En sus obras bibliográficas ella también traduce sus conocimientos históricos, bibliotecológicos y además su capacidad analítica de nuestra sociedad, reconociendo con lejanía algunos logros. La obra de Simons tiene un carácter indivisible, común a todos los bibliotecarios y bibliotecarias de profesión; su obra intelectual se refleja en su trabajo bibliotecario, y viceversa. Es por ello que el objetivo dual de este trabajo es por una parte demostrar la medida y la forma en que su legado llegó a ser parte fundamental del patrimonio institucional de la Biblioteca Pública, en calidad de políticas, reformas, reglamentaciones y concepciones, para por otra lograr sintetizar por primera vez con respaldo bibliográfico su influencia y recorrido profesional, históricamente poblados de enigmas.

\section{Metodología}

Como afirma Parada (2003), el rastreo de algunas "vidas bibliotecarias" es fundamental para configurar la historia de nuestra profesión. Estas vivencias permiten demostrar, desde la perspectiva del sujeto estudiado y su relación con el medio, el estado y la evolución disciplinar en un período dado de nuestra historia, pero también el desarrollo de nuestras instituciones y los cambios sustanciales en cuestiones de género y acceso público a la cultura.

Si bien existe una formada tradición de estudios bibliográficos como método de análisis biográfico en la bibliotecología argentina, se consideró que dicha metodología sería esquiva respecto de la riqueza extraíble del análisis de Simons por medio de otras alternativas, enfocadas más bien en su rol dual de bibliotecaria y su condición de mujer. Por esta razón, el abordaje de esta investigación sigue la metodología propuesta por Maack (Rodríguez Toajas, 2013; Tecuatl Quechol, 2016-2017) para el estudio específico de la mujer en bibliotecología, que propone el análisis de:

» Biografías de mujeres que participaron de la formación de la profesión (haciendo especial énfasis en el período de la primer inserción), el estudio de las razones que las llevaron a elegirla, sus trayectorias, su formación y lo que pensaban acerca de ésta.

" La participación en el desarrollo de los servicios bibliotecarios y de la extensión cultural.

》 La participación en el diseño de cualquier tipo de formación bibliotecaria.

" La participación en las asociaciones profesionales.

En palabras de Rodríguez Toajas (2013), y apoyándose en Hildebrand (2000) el principal problema para estudiar a estas primeras protagonistas femeninas es:

...Distinguir el discurso de la realidad, esto es, discernir cómo y en qué medida se corresponde la realidad con la retórica del relato hegemónico dominante que predomina y subyace en las fuentes primarias y secundarias y, en muchos casos, reproduce planteamientos androcéntricos (Rodríguez Toajas, 2013: 7).

El enfoque entre líneas es crucial para poder recuperar la información necesaria y acreditar la responsabilidad de los proyectos e innovaciones; esto significa, dilucidar por medio de declaraciones personales o por la atribución de responsabilidad de colegas. Hablamos entonces de fuentes informales, o bien que no corresponden a la propia 
oficialidad institucional. En el análisis de la trayectoria de Simons confluirán tres de los principios metodológicos de Maack (1982); el enfoque biográfico, los aportes a los servicios bibliotecarios realizados y las contribuciones a la formación bibliotecaria. De esta forma, se podrá medir su impacto personal, profesional e institucional.

\section{La búsqueda bibliográfica}

El análisis de la figura bibliotecaria, siguiendo a Romanos de Tiratel (2012), puede hacerse desde distintas perspectivas y remarcar alguna faceta específica de la personalidad estudiada. Sin embargo, en el caso de Simons, la regla ha sido la sedimentación. No existen estudios sobre su trayectoria, más que menciones al paso o de curiosidad por su origen extranjero. La solución metódica a este solapamiento del accionar de la mujer solo pudo ser encontrada en estos primeros estudios feministas sobre la bibliotecología.

Si bien el desarrollo de este tipo de investigaciones centradas en la mujer bibliotecaria comenzaron en la década de 1970 en los Estados Unidos, han tenido eco en nuestro campo hispanohablante. Por esta razón, la bibliografía fundamental ha sido la de Rodríguez Toajas (2013) por las sincronías a la hora de enfrentarse a la ausencia de estudios con perspectiva de género en nuestro campo, la compatibilidad de los análisis y la metodología propuesta, dadas las semejanzas socio-culturales con la España del mismo período. El aporte de Tecuatl Quechol (2016-2017), por ser un primer abordaje en nuestra región, particularmente en México, de las mujeres bibliotecarias. Y la relevancia de los trabajos de Maack (1982) y Hildebrand (2000) por ser los estudios fundacionales del campo en materia de género y de recurrencia obligatoria, por su aporte metodológico que aún sigue teniendo vigencia en este tipo de estudios que enfrentan dificultades con la carencia de fuentes primarias.

Desde el punto de vista de desarrollos locales y biográficos, fue necesario considerar las investigaciones en nuestro campo y su tratamiento de las personalidades bibliotecarias. Para esto, fue de gran utilidad el artículo sobre Josefa Sabor de Romanos de Tiratel (2012) por constituir un estudio reciente y de una bibliotecaria de un período posterior, el de Fernández (2005) sobre Luis Ricardo Fors y el de Parada (2003) sobre Couture de Troismonts por la cercanía institucional y jerárquica de los cargos ocupados con Simons, entre otros, que han servido de base para un enfoque biográfico con características bibliotecológicas.

\section{La búsqueda de fuentes}

Esta investigación demandó la consulta de una gran diversidad de fuentes, que permitieron la reunión y conexión de datos e información que eran transmitidos de forma oral pero de difícil comprobación. Cuando surgió el interés en investigar su figura, se buscó en el catálogo en línea de la Biblioteca Pública de la UNLP si existían libros de su autoría. Ante esto, la obra más relevante que se recuperó fue "Algunos aspectos de la biblioteconomía" (Simons, 1934), la cual compila una parte de sus obras publicadas en Argentina. Aun así, se extrajo poca información respecto de su vicedirección a partir de esta obra.

La conexión más significativa tuvo lugar cuando se consultó "Hanny Simons en mi memoria" de Angélica Costa Álvarez de Sapin (1966) y con algunas de las notas que se publicaron en los diarios La Opinión de La Plata y El Día, contando entre estas la carta de su hijo a la biblioteca durante la dirección de Couture de Troismonts. Sin el conocimiento biográfico de Simons difundido por Costa Álvarez de Sapin, sus atribuciones de responsabilidad y autoría, hubiese sido imposible continuar la investigación. 
De este artículo se pudo enlazar información de Konrad Simons, esposo de la bibliotecaria y el motivo de su presencia inicial en Argentina. Con esta base, se pudo continuar esta recopilación, a la cual hicieron un importantísimo aporte la Biblioteca de la Facultad de Ingeniería (UNLP) y el Centro de Documentación de la Inmigración de Habla Alemana. Estos últimos quienes, luego de dos años de búsqueda, pudieron brindarme acceso a todo el legado personal/familiar de Simons y su esposo, compilado por sus descendientes y donado a esta institución recientemente.

\section{La inmigración científica}

Simons era originaria como su marido de la ciudad de Elberfeld, Alemania, y residente en la ciudad de La Plata desde el año 1911. Desde un primer momento resulta visible la necesidad de comprender su actuación en el marco de una comunidad con valores y un entendimiento compartido de la realidad. Estos círculos académicos e intelectuales se veían diferenciados, además, por grados de afinidad cultural, ideológica, política, entre otros, existiendo así diferentes niveles concéntricos que, si bien visualizaban un mismo fin, son notoriamente distintos.

Así sucedía con la comunidad universitaria de la UNLP en sus inicios. Concebida como una universidad cimentada en las ciencias exactas y naturales, era lo que según Joaquín Víctor González demandaba la República Argentina, ya que favorecería su desarrollo técnico e industrial. Dicho proyecto educativo surgía de una visión de estado cuya herencia ideológica era primordialmente sarmientina, y surgía del seno de la misma clase acomodada y erudita que ya había entrelazado un importante sistema educativo y cultural. Fue fundamental, entonces, el poder contar con intelectuales conocedores de los modos, los debates más novedosos del momento y de las últimas tendencias de los círculos académicos vanguardistas de la época.

Al año 1909, la física es dominada por la academia alemana. Es entonces cuando González propone conseguir profesores extranjeros para el incipiente Instituto de Física de la UNLP. Luego de prepararse la búsqueda, al físico Emil Bose se le promete la gestión del Instituto y como dato no menor, que su esposa también sería contratada, lo que dejaría un antecedente, quizá, para el caso que nos convoca. Con la mediación de Bose, otros científicos alemanes arribaron a la universidad, entre los que se contaba Konrad Simons, un colaborador suyo del Instituto Tecnológico de Danzig.

A Konrad Simons se le asigna la misión de encabezar la organización de la carrera de Ingeniero Electricista en dicho instituto, con la garantía de que se le darán facilidades acordes a los costos de manutención de su familia. Luego de que se le asegurara dicha protección, viene al país junto con su esposa y sus hijos. Si bien su paso por la universidad fue breve por su muerte repentina, llegó a ser director interino del mencionado Instituto, logró dirigir cursos de electrotecnia y proyectar la instalación del instituto de la misma materia. Para entonces, la comunidad académica alemana ya era una parte importante de las instituciones universitarias locales, con personalidades como Roberto Lehmann Nitsche a cargo del Museo y Ricardo Gans en el Instituto de Física (Bibiloni, Civitarese y von Reichenbach, 2003; von Reichenbach y Bibiloni, 2012).

En 1919, un año después de la pérdida de su esposo y con dos hijos a cargo, Simons decide solicitar su ingreso a la Biblioteca Pública de la Universidad (Resolución No. 1875, 1919), donde rápidamente sería ascendida a Vicedirectora ad-honorem hacia 1921 (Comunicado interno Resolución del 27 de enero de 1921). Según Costa Álvarez la elección de la biblioteca no fue azarosa; en su ciudad de nacimiento se habría orientado al ámbito bibliotecario desde sus comienzos profesionales, llegando a ejercer en la Biblioteca Municipal de Elberfeld (Costa Álvarez de Sapin, 1966). Tal como Simons, 
Margrete Heiberg Bose, física y también viuda, tomaría la decisión de conducir una biblioteca universitaria. Bose decidió integrarse a la Biblioteca del entonces Observatorio Astronómico, cargo en el que estuvo alrededor de un año (Chicote y Göbel, 2011).

Caracterizado por sus innovaciones, este período de gestión comienza con el reconocimiento de la UNLP de su potencial para llevar adelante grandes cambios en la Biblioteca Pública, un hecho de destacable particularidad para los estándares vigentes. Si bien no se le otorga la dirección, desde el puesto de vicedirectora se le da el aval para introducir las modificaciones necesarias para poder adaptar la biblioteca a su carácter público, sin sacrificar el universitario. Si bien se puede aludir que esta decisión se basó en la utilización de sus conocimientos adquiridos en Alemania, constituye un importante reconocimiento a una mujer, considerando la predominancia masculina en los ámbitos intelectuales.

\section{La vicedirección}

Simons se incorpora a la biblioteca durante la gestión de Carlos Vega Belgrano, un periodista polifacético, fundador de múltiples diarios, asignado a su cargo por González en el año 1906. Era también, al momento, Vicepresidente de la Comisión Nacional de Bibliotecas Populares desde 1912 y sería convocado a presidir dicha institución en 1927, eventualidad que ella aprovecharía para dedicarle un artículo en el cual describe su trayectoria. Sobre Vega Belgrano se deduce la disposición de un conocimiento bibliotecológico adquirido durante el ejercicio de su cargo, y una visión de lo público que compartiría con Simons. Este hecho podría haber facilitado las innovaciones en la biblioteca que se llevaron a cabo con la guía o iniciativa la nueva vicedirectora, en especial en cuanto al préstamo domiciliario, acerca del cual las bibliotecas populares, el otro dominio de Vega Belgrano, tenían décadas de experiencia para entonces (Planas, 2017; Simons, 1932).

Durante este período de vicedirección, es decir, el que coincide con la dirección de Vega Belgrano (1907-1930), Simons dedica sus esfuerzos a las innovaciones en la biblioteca. Como bibliotecaria, desarrolla un catálogo de biografías (Costa Álvarez de Sapin, 1966). Ya al asumir la vicedirección en 1921, Costa Álvarez y Palcos atribuyen a su trabajo la reglamentación del préstamo domiciliario, implementado finalmente el 11 de marzo de 1926, y la creación del primer sistema de clasificación de la biblioteca pública, el mismo año. El 27 de enero de 1921, el presidente de la UNLP, Carlos F. Melo dicta y comunica en la misma disposición en la que queda explicitado el contrato de Simons, que la finalidad de su ascenso como vicedirectora era la de encabezar un proyecto de transformación que implicaría la apertura al público a partir del 1 de febrero de ese mismo año, que por entonces se restringía a la sala de lectura:

Siendo indispensables los servicios de la Biblioteca,... habiéndose ausentado, según resulta del precedente informe, los empleados de la Biblioteca sin tener en cuenta sus obligaciones especiales, ni la inauguración de los servicios nuevos que se comprometió la Universidad desde su fundación y que no ha prestado; (...) y estando conforme con ello la señora de Simons en dedicar toda su acción para la reorganización de ese establecimiento; (...) el Presidente de la Universidad decreta: Art. 1: Desígnase Vicedirectora de la Biblioteca de la Universidad de La Plata ad-honórem a la señora Hanny Simons. Art. 2: La vicedirectora procederá de acuerdo con el Director a la reorganización de la Biblioteca y su habilitación para que funcione para el público desde el 1 de Enero de 1921 (...) (Comunicado interno Resolución del 27 de enero de 1921). 
Desde la dirección de Augusto Belín Sarmiento, quien proyectó una biblioteca circulante, la idea de que los libros pudiesen ser extraídos de la biblioteca había sido descartada, en favor de una concepción elitista de la institución bibliotecaria (Dorta, 2017). Mucho menos pensada sería, hasta entonces, la posibilidad de que pudiese facilitarse la lectura a todos los vecinos de la ciudad hasta sus propios hogares. La reglamentación del préstamo domiciliario pudo ser establecida tras vencerse "reticencias históricas" (Costa Álvarez de Sapin, 1966) a la circulación del material, que se habían originado bajo la dirección erudita de Fors, desde 1898 hasta 1907 (Fernández, 2005). La marcada inclinación del primer director de la entonces Biblioteca provincial a la reunión y la preservación bibliográfica llegaba al punto de disponer un vigilante de servicio en la sala de lectura de la Biblioteca Pública de la Provincia. Puede así visualizarse la contraposición de dos posturas en pugna -por un lado, la preservacionista, y por otro, la aperturista-. Al respecto, Palcos decía:

La respectiva reglamentación -proyectada por la vicedirectora, señora Hanny Simons-establece que para este beneficio el lector deberá sacar su tarjeta de tal y comprobar su calidad de vecino platense; se le presta hasta tres libros a la vez y por siete días... Hay personas aún hoy alarmadas del sistema: lo suponen incompatible con la psicología criolla. Los resultados de ocho años de práctica desvanecen muchas presunciones contrarias. Desde este punto de vista el ensayo ostenta importancia social. Desde luego se llevan perdidos al presente varios centenares de libros y otros son devueltos en condiciones poco recomendables; pero si atendemos a una queja del doctor Fors cuando los libros eran exclusivamente consultados en la sala de lectura, abundaban los lectores inescrupulosos que ajaban sus hojas, las llenaban de dibujos, arrancaban sus láminas o deterioraban las colecciones de diarios y revistas. Fuerza es admitir, por lo tanto, que el nivel medio de los lectores ha mejorado (Palcos, 1934: 17).

Para visualizar las dimensiones y la significancia de esta modificación del servicio bibliotecario, las cifras pueden resultar de gran ayuda, tal como se observar en el crecimiento exponencial de la cantidad de lectores, tomando como referencia desde 1921 hasta 1944 (Cuadro 1). Se ha de tener en cuenta el salto significativo en el año 1926; es entonces cuando se completa la reglamentación y es aprobada por la UNLP el 11 de marzo de ese mismo año.

\begin{tabular}{cc}
\hline Año & Cantidad de lectores \\
\hline 1921 & 5.278 \\
1925 & 8.694 \\
1926 & 26.264 \\
1944 & 121.189 \\
\hline
\end{tabular}

Fuente: Llovet, 1967

Cuadro 1. Cantidad de lectores (1921-1944)

El debate entre la concepción universitaria y la pública de la Biblioteca, lejos de extinguirse con la reglamentación del préstamo domiciliario, continuó como polémica hasta avanzado el siglo XX (Bossié, 2006). Bajo la dirección de Couture de Troismonts, el nombre de la Biblioteca Pública volvería a la forma de sus inicios: "Biblioteca Central de la Universidad". A esta transformación sucedió el recorte, además, del préstamo al público en el año 1967; los argumentos de Fors volvieron a tener predominancia. En medio de un gran debate público llevado a cabo por los usuarios de la Biblioteca a través del diario local El Día, destacaría una carta de Hellmut Simons ${ }^{2}$, hijo de Simons. En ella, argumentaría: 
Por razones obvias han merecido mi preferente atención las recientes publicaciones relacionadas con las modificaciones introducidas en el régimen de la Biblioteca Pública de la Universidad (...), modificaciones que, implican desde ya suprimir una parte de su nombre, pues ha dejado de ser pública. No quiero agregar nuevos argumentos a los muchos que se han dado en sus columnas contra la supresión del servicio de préstamo a domicilio, verdadero atentado a la cultura popular y medida injustificada en todo sentido. (...) Quiero destacar tan solo que, - salvo que un nuevo convenio entre el Gobierno de la Nación y el de la Provincia de Buenos Aires no se haya dado a publicidad- el proceder del nuevo Director de la Biblioteca, atenta contra las disposiciones de la Ley Convenio que dio origen a la creación de la Universidad(...) (Simons, 1968).

Simons, en su artículo "La biblioteca vista por dentro" (Simons, 1932b), argumenta su postura favorable al préstamo domiciliario en el acto de brindar facilidades para la lectura, siempre que este servicio sea acompañado de una serie de controles rigurosos que impidan el deterioro del patrimonio.

En el camino de la Biblioteca hacia su nuevo perfil, la reglamentación del sistema de préstamos domiciliarios constituía el horizonte y así poder convertir la Biblioteca Central en Biblioteca Pública. Sin embargo, era evidente que el sistema de clasificación vigente, el establecido por Fors en el año 1906, pensado para un modelo de biblioteca erudita, iba a verse superado por los flujos del fondo documental, en plena expansión. Surgiría la necesidad entonces de un sistema de organización capaz de soportar una gran proporción de transacciones diarias, de fácil aprendizaje para los responsables de la atención al público. Aquí tomaría preponderancia uno de los primeros debates bibliotecológicos de nuestro país en cuanto a la organización del conocimiento.

Hasta entonces la Guía para los trabajos de clasificación y catalogación bibliográfica ideada por Fors regía el orden bibliográfico. Ésta es una adaptación del sistema pensado por Paul Groussac para la Biblioteca Nacional (Biblioteca Nacional de la República Argentina, 1893), compuesto de 5 secciones que tenían la intención de ser accesibles para los lectores. Se basaba a su vez en el Manuel du libraire et de l'amateur de livres de Brunet promovido en el país por Ernesto Quesada (Arcella, Bizzotto y Zeballos, 2009). Groussac compartía con Simons un punto decisivo en la concepción de un sistema de organización; la sencillez. Por esta misma razón explicaba que el mejor modo de distribución de la bibliografía tenía que cumplir tres condiciones fundamentales: accesibilidad, elasticidad y racionalidad. Lacónicamente, afirmaría: "un catálogo es por excelencia una obra de vulgarización” (citado por Arcella, Bizzotto y Zeballos, 2009: 25).

La catalogación, más allá de la descripción bibliográfica, contenía a la organización y la definía según múltiples parámetros según la conveniencia y la practicidad. Era la herramienta definitiva para garantizar una biblioteca democrática, ya que la ausencia de formación técnica en el personal (es pertinente recordar las observaciones de Cónsole en 1931) y la predominancia del saber empírico demandaba sistemas de clasificación puestos en función del servicio ágil y con una rápida vinculación a los catálogos (Arcella, Bizzotto y Zeballos, 2009).

Resulta evidente la razón por la cual la "Guía de clasificación" de Fors fue mejorada antes de ser descartada por un sistema decimal. Esta se dividía en 12 secciones, 8 de materias de estudio y 4 auxiliares. El sistema que lo suplantara, publicado en 1926, sería diseñado a su vez sobre las divisiones temáticas que Fors ideó, que se conservaron con pequeñas modificaciones terminológicas y la inserción de la sección XI, Cartografía, dentro de Geografía. Estas nuevas categorías llevarían ahora, a modo de subdivisión, dos letras identificatorias (una letra característica y una subdivisión) y un número. El determinante de la nueva metodología de ordenamiento de los libros 
sería lo que Simons definiría como el Numerus Currens, de acuerdo al cual los libros son ordenados en las estanterías de acuerdo a su ingreso en la biblioteca. A favor del mismo aduce que permite saltear los espacios que demanda la clasificación decimal entre las temáticas y una combinación útil con la modalidad mnemotécnica de la utilización del abecedario. Simons consideraba el modelo de organización decimal de Dewey y el Decimal como "impracticables" y "utópicos", lo que debe entenderse en su contexto histórico, es decir, estanterías vedadas al público, practicidad empírica y un servicio demandante. En razón de esto, afirmaba:

(...) El bibliotecario actual tiene, ante todo, el deseo de mejorar su sistema, y mejorarlo será siempre buscar su simplificación, porque el sistema más simple, el que menos tiempo exige para la búsqueda de los libros es el que mejor cumple con los principios de la biblioteconomía, y el que, en forma más satisfactoria, deja libre en las horas del trabajo el tiempo necesario para las demás tareas (Simons, 1934: 24).

En la elaboración del sistema de clasificación no solo participó el personal de la biblioteca: Costa Álvarez asegura que para elaborar este sistema se convocaron a distintas personalidades platenses, con experticia cada uno de ellos en la temática específica requerida. Según la autora, y por la presencia destacada del círculo académico alemán, podemos confirmar que estuvo detrás de estas participaciones. Así, por ejemplo, se convocó para elaborar la sección de Ciencias Físicas y Matemáticas, al físico Ricardo Gans (antiguo compañero de su difunto esposo); para la sección Filosofía, Educación y Religión, al presbítero Leandro B. Astelarra, para la sección de Ciencias Naturales y Ciencias Médicas, al naturista Roberto Lehmann Nitsche y al doctor Moises Jeréz respectivamente. Entre otros como César Díaz Cisneros, Rómulo Carbia y Agustín Millares Carlo, de los cuales no se dispone mayor información de su colaboración. Fiel a la trayectoria institucional, Simons reconocía en el modelo de Fors y sus divisiones, las bases de la nueva organización del fondo bibliográfico de la Biblioteca, sobre lo cual afirmaba “(...) en bibliotecología es una norma que tiene importancia de ley, construir sobre lo existente, perfeccionándolo (...)” (Simons, 1934: 24).

Quedarían entonces definidas las nuevas áreas, numerando básicamente las subdivisiones alfabéticas como lo muestra el Cuadro 2:

\begin{tabular}{|l|l|}
\hline $\begin{array}{l}\text { I: Ciencias Naturales. Ciencias Médicas. } \\
\text { Letra característica: N } \\
\text { Subdivisiones. Na, Nb, Nc, Nd, Ne, Nf, Ng, Nh }\end{array}$ & $\begin{array}{l}\text { VII: Sociología y Ciencias Económicas } \\
\text { Letra característica: S } \\
\text { Subdivisiones: Sa, Sb, Sc, Sd, Se, Sf, Sg, Sh }\end{array}$ \\
\hline $\begin{array}{l}\text { II: Ciencias Físicas y Matemáticas } \\
\text { Letra característica: M } \\
\text { Subdivisiones: Ma, Mb, Mc, Me, Mf, Mg, Mh }\end{array}$ & $\begin{array}{l}\text { VIII: Derecho } \\
\text { Letra característica: D } \\
\text { Subdivisiones: Da, Db, Dc, Dd, De, Df, Dg, Dh, } \\
\text { Di, Dk, DI, Dm }\end{array}$ \\
\hline $\begin{array}{l}\text { III: Arte } \\
\text { Letra característica: A } \\
\text { Subdivisiones: Aa, Ab, Ac }\end{array}$ & $\begin{array}{l}\text { IX: Sección Oficial } \\
\text { Letra característica: O } \\
\text { Subdivisiones: O. (Entidad Gubernamental) }\end{array}$ \\
\hline $\begin{array}{l}\text { IV: Literatura } \\
\text { Letra característica: L } \\
\text { Subdivisiones: La, Lb, Lc, Ld, Le, Lg, Lf, Lh, Li, } \\
\text { Lk, LI }\end{array}$ & X: Diarios y Periódicos \\
\hline $\begin{array}{l}\text { V: Historia, Geografía y Ciencias Afines } \\
\text { Letra característica: H } \\
\text { Subdivisiones: Ha, Hb, Hd, He, Hg, Hi, Hk, Hm, } \\
\text { Hn. Ho, Hp }\end{array}$ & XI: Autógrafos y Retratos \\
\hline $\begin{array}{l}\text { VI: Filosofía, Educación y Religión } \\
\text { Letra característica: F } \\
\text { Subdivisiones: Fa, Fb, Fc, Fe, Fd }\end{array}$ & \\
\hline
\end{tabular}


3. Este documento se encuentra en el Catálogo web de la Biblioteca Pública con el nombre de "Vistas de bibliotecas (44 tarjetas postales disponible)" y disponible en el fondo general de esta biblioteca bajo la signatura Lk-117o.

4. Su hija Ingeborg Simons fue también contratada por la biblioteca durante este período, en el cual llevó a cabo, ya durante la dirección de Alberto Palcos

(1930-1946) y con la colaboración de Ricardo Levene, la elaboración del Catálogo de periódicos sudamericanos, publicado en 1934.
Así, por ejemplo, Lk es, dentro de la sección Literatura, la sección de Bibliografía, donde se incluye la subcategoría "Bibliotecas y Biblioteconomía". A un documento recién ingresado de esta temática, debería asignársele estas dos letras seguidas de un número que se correspondería al último disponible de esa subdivisión. Por ejemplo, si el anteúltimo ejemplar adquirido fuese "Lk-506", el recién llegado sería el "Lk-507". En el año 1936 saldría la última versión del sistema, con algunas modificaciones ampliatorias relacionadas al uso de los ficheros y nuevas subdivisiones. Esta sería la última versión del sistema de clasificación, que aún hoy está en uso.

Durante su administración tuvieron lugar otros hechos y obras que se encuentran ligados a su persona. Ejemplo de esto son las listas de libros entrados, originalmente denominados con esa intención "listas de libros más leídos". Estas, como listados ordenados y sistemáticos, no fueron continuadas regularmente. Por otra parte, las autoridades de la universidad le encomiendan visitar bibliotecas europeas, con el fin de conocer estilos arquitectónicos y de servicio que permitieran mejorar los ofrecidos en la Biblioteca Pública y aportar nuevas ideas en el marco de la construcción de su edificio actual, establecido en 1934. Esta información puede corroborarse, por un lado, con las sucesivas licencias con goce de sueldo que se le otorgan con "motivo de trasladarse al extranjero" para "visitar las bibliotecas de ese país [Alemania] y presentar un informe" (Comunicado interno Resolución del 6 de junio de 1921) durante su gestión. Otra prueba documental es la compilación de postales de bibliotecas de Europa occidental que se encuentra en el fondo bibliográfico de la Biblioteca Pública, con su firma y anotaciones manuscritas en alemán, en algunos casos 3,4. En 1933 dirige una última gran transformación en la biblioteca. Esto fue la centralización del catálogo de la biblioteca, experiencia a partir de la cual, probablemente, consideraría la re-edición del primer sistema de clasificación en el año 1936 (Aguado de Costa, 2006).

Al observar el plano de la transformación que Simons impulsó en la Biblioteca Pública, se plasma una red de ampliaciones o mejoras de servicios que van, entonces, desde la forma de comprender y ordenar el acervo bibliográfico, la forma de recuperar el ejemplar particular, los derechos del usuario a la lectura y, finalmente, la renovación total del trabajo del bibliotecario. Sin dudas, hechos que resultaron de una planificación integral y no una serie de logros desconectados y acontecidos por azar bajo su conducción.

\section{El recorrido intelectual y la influencia de Simons}

En este apartado se hace visible un punto de análisis que, quizás, no se encuentre totalmente representado en la metodología de Maack (1982), pero que se hace necesario. El estudio de la producción intelectual perse, como sí lo notara Rodríguez Toajas (2013) al descubrir la incipiente producción intelectual femenina de las primeras generaciones de bibliotecarias, todas relacionadas con las bibliotecas y los servicios bibliotecarios, que de compartir características con las de Simons, demuestran, contra las creencias sociales de la época, que las mujeres mucho podían y tenían que aportar, y que de hecho lo hacían, opinando sobre los espacios en que podían llegar a desempeñarse en su tiempo.

En su período de administración, Simons se dedica también a estudiar la teoría biblioteconómica; publica artículos de la materia en los diarios El Día, El Argentino, diversas revistas científicas y también sus dos libros (Simons, 1932, 1934). En el período que se desempeñó como vicedirectora, Costa Álvarez acota que era percibida como maestra por sus compañeros, siendo su legado directo al personal múltiples conocimientos biblioteconómicos que hubiesen sido imposibles de adquirir de otra forma. Aguado 
de Costa denomina este hecho una "escuela no oficial" en la cual impartió saberes de forma permanente, una "capacitación en servicio" gracias a la cual fue posible la futura existencia de la Escuela de Bibliotecarios en la biblioteca. La conexión que representa Simons con el saber y el desarrollo bibliotecológico de Europa es fundamental; este tipo de contactos interculturales habilitaba la toma de conocimiento de realidades sociales alternativas y, como queda demostrado en el trabajo de Tecuatl Quechol (2016-2017) sobre las primeras bibliotecarias mexicanas, el intercambio internacional era un importante factor de empoderamiento femenino local, la toma de conciencia respecto de las posibilidades profesionales, la apertura de nuevos espacios laborales y el derribamiento de los mitos alrededor de las capacidades de la mujer.

Lo primero que se percibe al leer sus escritos es un perseverante énfasis en la transmisión de conocimientos. Sus obras presentan una estructura didáctica y de lectura ligera, siguiendo la regla de hacer accesible la idea que quiere comunicar. Demuestra conocimiento de los detalles de la historia de la profesión, y lo plasma en artículos que tratan de las bibliotecas y los libros a lo largo de la historia. Ejemplo de esto son los artículos publicados en el libro compilador Bibliotecas y Bibliotecarios (1932). En él se incluyen Goethe y las bibliotecas (1926), Erasmo y sus impresores (1925), La bibliomanía en la edad media. A propósito de Tito Livius (1924), Primeros pasos de la Imprenta. Algo sobre incunables (1924). Revela en ellos buena parte de su saber respecto de la cultura bibliotecaria y librística de Europa, dando a conocer, por ejemplo, un Goethe vinculado con la labor bibliotecaria, el tratamiento del libro en la edad media, la historia de la imprenta y la cuestión de los incunables a través del tiempo. Respecto de estos últimos, compila además los resultados de la investigación realizada por Lázaro Galdiano (1925) respecto de los incunables bonaerenses, en los que hace mención de las publicaciones realizadas por la Imprenta de los Niños Expósitos (Simons, 1932c).

Analizó también el funcionamiento de la biblioteca de la extinta Liga de las Naciones, de la cual destacó el "reconocimiento más amplio de la capacidad intelectual de la mujer que recordarse pueda" (Simons, 1932a: 73), en relación a que en dicha biblioteca solo trabajaban mujeres y estas eran dirigidas por una bibliotecaria, la estadounidense Florence Wilson. Otros artículos fueron motivados por sus análisis de la Institución Carnegie y la Comisión Internacional de Cooperación Intelectual, ambos casos vinculados con el favorecimiento de la paz y del intercambio científico e intelectual internacional, tópicos recurrentes en sus análisis.

Dos temas centrales en su obra eran el lector y el bibliotecario; partes fundamentales de la biblioteca, a la que entendía como una institución viva, esto es, orgánica. En estos escritos vierte su experiencia y sus esfuerzos intelectuales por comprender la realidad local. Está claro que el pasaje de una biblioteca exclusiva a una biblioteca abierta al público general generaba múltiples interrogantes respecto de lo que se debía ofrecer o qué debía esperarse del lector, teniendo en cuenta que ya no se limitaría a estudiantes de la universidad o eruditos sino también a cualquier "vecino de la ciudad". En el afán de visualizar una ciudadanía ideal o bien, proyectando una serie de condiciones privilegiadas a toda la población, no es difícil encontrar entre la bibliografía contemporánea contradicciones en las expectativas sobre el público general y la realidad social. Un ejemplo de estas polémicas puede verse en su artículo enviado a la revista Valoraciones, Organicemos nuestra cultura: el lector(Simons, 1932d), en el cual refuta que en la ciudad de La Plata sea común la lectura en idioma francés, interviniendo en una polémica de la publicación. Su visión sobre la situación cultural argentina procuraba ser realista. A Simons le inquietó el desinterés respecto de la cultura popular, recelo que surge de su paulatina toma de conocimiento del estado de la lectura en el país, hecho que destaca como un obstáculo inhabilitante para las bibliotecas, que no obtienen el reconocimiento y apoyo necesario para cumplir sus funciones. Al respecto, comentaba: 
Si no hay lectores, no hay necesidad, ni posibilidad, de progresar y perfeccionar nada, y si los libros y revistas, en una biblioteca, no se leen, para qué y por qué se van a comprar más? Sería un archivo vivo (Simons, 1932d: 129).

Con respecto al rol del bibliotecario, tampoco se privaba de intervenir en el debate exponiendo un lugar común que no ha dejado de existir, necesariamente: "Se piensa en que es el empleo más cómodo, que el poner un libro al alcance del público es un trabajo reducido y limitado, que puede hacer cualquier persona", afirmaba (Simons, 1932: 63-64).

En contraposición, su imagen del bibliotecario estaría fuertemente marcada por un perfil servicial y entregado, desde el cual se puede entender el porqué de su sorpresa ante el interrogante anterior. En razón de discutir esta noción, describía al público lector de los periódicos, paso por paso, todas las tareas realizadas al interior de la biblioteca, incluyendo aquellas que al día de hoy consideramos necesariamente ajenas al usuario, como son los procesos técnicos.

(...) El bibliotecario debe ser el alma y la fuerza motriz de esta máquina, que le dá vida, que nunca debe hacer dificultades formales a sus lectores, que deberá salvar todas las que surjan, que siempre debe estar a la disposición de todos y de cada uno, porque el lema de los bibliotecarios del mundo entero es: "ich dien" (yo sirvo) (Simons, 1932: 68).

Consideraba también que se exigía del bibliotecario el ser una fuente de conocimiento universal viviente, el poseer un saber general importante. De otra forma, aseguraba, estaría desnudo ante un público que lo sobrepasaría con sus preguntas. Esta noción expone su impronta europea-continental, es decir, enciclopedista. Por ello destacaba en sus artículos la importancia del conocimiento general; Besio Moreno diría, al prologar la compilación de los mismos:

(...) La biblioteca como instrumento de acción, no ha de ser el instituto parasitario de una sabiduría expectante, sino el agente vivificado de una poderosa fuerza centrífuga que trasciende más allá de sus muros y portales, que deben ser perforados y atravesados dilatadamente, como perfume que acude por doquier y se hace sentir por aquellos que no lo ansían. Así nos enseña a comprender las bibliotecas la autora (Simons, 1932: 9).

Por otra parte, la vida profesional de Simons es simultánea a un período de grandes transformaciones en el campo de la disciplina bibliotecológica. En concordancia a las necesidades de su tiempo, la necesidad de hacer "del bibliotecario un profesional" se hacía imperiosa ante el crecimiento del público lector y del caudal de publicaciones, demandando habilidades y conocimientos específicos para gestionar organizaciones en rápido crecimiento. Es así como, orientándose gradualmente a una conceptualización científica integral, en Argentina tuvieron lugar los primeros debates disciplinares, que buscaban definir el significado de la Biblioteconomía.

Este era el nombre que definía el estudio de la organización y administración de las bibliotecas, un campo del conocimiento que, a diferencia de lo que comprendemos en la actualidad como Bibliotecología, era extraído casi por completo de la práctica laboral y cuyo desarrollo buscaba la mejora exclusiva de esta. Implicaba el estudio de la biblioteca y sus funcionalidades, también para conocer prácticas pasadas y para hacer visible una evolución de la profesión a lo largo del tiempo. Por esta razón la visión de Simons incluía la historia del libro, de la imprenta, del comercio del libro, la legislación, investigación de los manuscritos, arquitectura de los edificios de bibliotecas y sus instalaciones, las clasificaciones, la administración, la adquisición 
de conocimientos generales y básicos de todas las ciencias. Al tiempo que el oficio se encaminaba hacia su profesionalización, decía; “...se ha llegado a considerar la biblioteconomía ya no como un arte, sino que muchos le dan la categoría de ciencia" (Simons, 1934: 1).

En la primera década del siglo XX en la Argentina es posible contar numerosos intentos de creación de una carrera de bibliotecarios, llevado a cabo por distintas personalidades en ocasiones no relacionadas directamente con la profesión. Estos intentos se materializaban en proyectos, implementados o no, de cursos y escuelas de bibliotecarios. Era una necesidad que remarcaba no solo Simons, sino toda la intelectualidad vinculada a la disciplina bibliotecaria, por ejemplo, Pablo Pizzurno, Federico Birabén, Ponciano Vivanco, Ricardo Rojas, Alfredo Cónsole, entre otros. Simons decía al respecto:

Para la organización de las bibliotecas, falta una cosa, en este país, que no falta en Europa ni en Estados Unidos. Ello es el estudio de la biblioteconomía; es la carrera del Bibliotecario. (...) Es que el defecto principal reside aquí, en que las bibliotecas son poco consideradas, y esto solamente puede remediarlo el lector (Simons, 1932d: 129).

Una importante indagación general sobre la Biblioteconomía es la que realiza en Algunos aspectos de la biblioteconomía (Simons, 1934), un ensayo que, en su estilo pedagógico, se explaya sobre los puntos y debates más importantes del campo. Dicha publicación le merecería un reconocimiento de Frédéric Finó (1944), en su conferencia Los estudios del bibliotecario, brindada en el año 1943 en el Museo Social Argentino, con motivo de la creación de un curso de bibliotecología general.

Además de las publicaciones en español, se conocen contados artículos que Simons publicó en revistas alemanas, y que refieren a la profesión y a su ejercicio en nuestro país. Entre estos se cuentan Bibliothekwessen in Argentinien (1926a) y Die Quesadas Als Förderergeistiger beziehungen zwischen argentinien und deutschland (1933-1934). Un caso aparte sería Breve noticia sobre el conde Keyserling (1926b)s.

\section{Reflexiones finales}

A modo de conclusión, es pertinente remarcar el carácter estructural de las obras y proyectos de Simons para poder comprender la dimensión institucional de la Biblioteca Pública, hecho que hace notoria su falta de representación (o bien, subrepresentación) alrededor de sus logros, sea en publicaciones periodísticas u oficiales. La trayectoria de Simons evidencia el caso de incontables mujeres de principios del siglo pasado que canalizaron tareas de reestructuración centrales en las bibliotecas sin estar presentes en el primer plano. Por esta causa, y por las limitaciones académicas conocidas que se imponían al género femenino por entonces, se demuestra la importancia del análisis biográfico-bibliotecológico por medio del estudio del legado profesional y la fusión de este con la vida de la institución, transformando y haciendo a esta en su desarrollo. En su nota de renuncia, declararía:

En los últimos años, la pasión política que siempre consideré debía permanecer al margen de nuestras tareas determinó conflictos de orden individual que traté de evitar o suavizar en cuanto de mi función dependía. Creo que a esta norma y a mi criterio de la función social de la Biblioteca que sustentara desde los días de la implantación del servicio de préstamo a domicilio, se debió que no obstante los profundos cambios habidos, pudiera continuar en mis tareas a entera satisfacción de los distintos Interventores y Directores que se sucedieron al frente de la Institución... (Simons, 1949: 2).
5. En términos generales, Bibliothekwessen in Argentinien (1926a) se desarrolla como un racconto didác tico de nuestra historia bibliotecológica, desde los inicios coloniales hasta la época de la autora. Die Quesadas Als Förderer geistiger beziehungen zwischen argentinien und deutschland (1933-1934) es un análisis del carácter bibliófilo de la figura de Vicente G. Quesada y su obra en Argentina. Por último, la Breve noticia sobre el conde Keyserling (1926b) es un análisis biográfico del sujeto en cuestión. 
6. Véase "La Biblioteca y la cultura pública" (1935)
Esta herencia institucional de la Biblioteca Pública de la UNLP significó, entre otros, que el servicio al público se sostenga hasta nuestros días, y, cuando fuese eliminado, regrese cual derecho adquirido y reclamado por parte de la ciudadanía platense en su periódico más popular. Otro legado de su administración es el pensamiento comprensivo de la biblioteca como una institución cultural que brinda un servicio social, no como favor sino como deber. Una organización que favorece la cultura y el desarrollo por medio del apoyo a la educación, integrando la apertura de los préstamos a toda la población y a su organización del fondo documental priorizando el servicio al público, en detrimento de la biblioteca erudita que tiende al cercenamiento antes que a la difusión. Nociones de progreso que remiten, finalmente, a la filosofía y proyección universitaria de Joaquín Víctor González $(1935)^{6}$.

Finalmente, su retrato ocupó las paredes del departamento de Procesos Técnicos de la Biblioteca Pública el suficiente tiempo como para volverse objeto de curiosidad e interrogación. Hecho admirable y a la vez exclamativo, un llamado a la reivindicación profesional que, como seguramente tantos otros, merece ser tenido en cuenta. 


\section{Referencias bibliográficas}

》 Aguado de Costa, Amelia. 2006. La Biblioteca Pública de la Universidad Nacional de La Plata, a través del tiempo. En Palabra Clave. Edición Especial 2006, 215-225.

"Arcella, Elvira, Mabel Bizzoto e Ignacio Zeballos. 2009. Biblioteca Nacional: los procesos técnicos en el Centenario. En Encuentro Nacional de Catalogadores (20: 2009: Buenos Aires). Buenos Aires: Ediciones Biblioteca Nacional. p. 1928.

»Bibiloni, Aníbal Guillermo, Osvaldo E. Civitarese y María Cecilia von Reichenbach. 2003. Una pionera de la física en la Argentina: Margrete Heiberg de Bose. En Anales AFA. Vol. 14, no. 1, 7-10.

»Biblioteca Nacional de la República Argentina. 1893. Catálogo metódico de la Biblioteca Nacional, seguido de una tabla alfabética de autores. Buenos Aires: P. E. Coni é hijos.

"Bossié, Florencia. 2006. Historias en común: censura a los libros en la ciudad de La Plata durante la última dictadura militar (1976-1983). La Plata: Universidad Nacional de La Plata. 130 p. Tesis de grado.

»Chicote, Gloria B. y Barbara Göbel, eds. 2011. Ideas viajeras y sus objetos: El intercambio científico entre Alemania y América austral. Madrid: Iberoamericana; Frankfurt: Vervuert.

»Cónsole, Alfredo. 1954 (1931). Hagamos de bibliotecario un profesional. 7a. ed. Buenos Aires: El Ateneo.

" Costa Álvarez de Sapin, Angélica. 1966. Hänny Simons en mi recuerdo. En Revista de la Universidad. Vol. 20-21, 396-399.

»Dorta, Ayelén. 2017. Espacios bibliotecarios de lectura: constitución y desarrollo de la Biblioteca Pública de la Provincia de Buenos Aires en La Plata (1884-1891). La Plata: Universidad Nacional de La Plata. Facultad de Humanidades y Ciencias de la Educación. 94 p. Tesis de grado. <http://www.memoria.fahce.unlp.edu.ar/ tesis/te.1651/te.1651.pdf> [Consulta: 28 febrero 2020].

» Fernández, Stella Maris. 2005. Luis Ricardo Fors: polígrafo y bibliotecario (creador de la colección cervantina de la Biblioteca Pública de La Plata). Buenos Aires: Sociedad de Investigaciones Bibliotecológicas.

» Finó, Frédéric. 1944. Los estudios del bibliotecario. Buenos Aires: Imprenta Coni.

»González, Joaquín V. 1935. La Biblioteca y la cultura pública. En González, Joaquín V. Obras completas, XVI. Buenos Aires: Universidad Nacional de La Plata. p. 139-159.

» Gutiérrez, Leandro H. y Luis Alberto. Romero, 1989. Sociedades barriales, bibliotecas populares y cultura de los sectores populares: Buenos Aires, 19201945. En Desarrollo económico. Vol. 29, no. 113, 33-62.

» Hildebrand, Suzanne. 2000. Library feminism and library women's history: activism and scholarship, equity and culture. En Libraries and Culture. Vol. 35, no. $1,51-65$.

»Lázaro Galdiano, José. 1925. Los Incunables bonaerenses. Madrid: La España moderna. 
»Llovet, Carlos. 1967. Biblioteca Pública de la Universidad. Historia. Los Hechos. Los hombres. Sus vidas. Documentos oficiales. La Plata: Biblioteca Pública de la Universidad Nacional de La Plata.

»Maack, Mary Niles. 1982. Towards a history of women in librarianship: a critical analysis with suggestions for further research. En Journal of Library History. Vol. 17, no. 2, 164-185.

»Palcos, Alberto. 1934. Síntesis sobre la fundación y organización actual de la biblioteca. En Boletín de la Universidad Nacional de La Plata. Vol. 18, no. 4, 5-36.

»Parada, Alejandro E. 2003. Semblanza de Roberto Couture de Troismonts, 19182001 (una contribución a su biobibliografía). En Infodiversidad. Vol. 5, 117-134.

»Planas, Javier. 2017. Libros, lectores y sociabilidades de lectura: una historia de los orígenes de las bibliotecas populares en la Argentina. Buenos Aires: Ampersand.

»Rodríguez Toajas, Alba. 2013. Mujeres y trabajo: la feminización de la profesión bibliotecaria. Madrid: UCM. 55 p. Tesis Máster Universitario.

"Romanos de Tiratel, Susana. 2012. El legado bibliográfico-bibliotecológico de Josefa Emilia Sabor (1916-2012). En Información, Cultura y Sociedad. No. 27, 11-33. <http://revistascientificas.filo.uba.ar/index.php/ICS/article/view/681> [Consulta: 28 febrero 2020].

"Simons, Hanny. 1926a. Bibliothekwessen in Argentinien. Separata de Minerva. Vol. 2, no. 2/3, 44-47.

» Simons, Hanny. 1926b. Breve Noticia sobre el conde Keyserling. En Sagitario. Vol. 1, no. 5, 206-209.

»Simons, Hanny. 1932. Bibliotecas y bibliotecarios. La Plata: Olivieri y Domínguez.

"Simons, Hanny. 1932a. La biblioteca de la Liga de las Naciones. En Bibliotecas y bibliotecarios. La Plata: Olivieri y Domínguez. p. 69-79.

»Simons, Hanny. 1932b. La biblioteca vista por dentro. En Bibliotecas y bibliotecarios. La Plata: Olivieri y Domínguez. p. 130-146.

"Simons, Hanny. 1932c. Los incunables bonaerenses por J. Lázaro. En Bibliotecas y bibliotecarios. La Plata: Olivieri y Domínguez. p. 56-59.

"Simons, Hanny. 1932d. Organicemos nuestra cultura: El lector. En Bibliotecas y bibliotecarios. La Plata: Olivieri y Domínguez. p. 125-129.

"Simons, Hanny. 1933-1934. Die Quesadas als Förderer geistiger Beziehungen zwischen Argentinien und Deutschland. Separata de Ibero-amerikanisches Archiv. Vol. 7, no. 2, 182-187.

»Simons, Hanny. 1934. Algunos aspectos de la biblioteconomía. La Plata: Olivieri y Domínguez.

"Simons, Hellmut. 1968. La Biblioteca Pública de la Universidad. En El Día. 9 de marzo, 6.

»Tecuatl Quechol, María Graciela. 2016-2017. A cien años de la participación de la mujer en la bibliotecología en México. En Bibliotecas y Archivos. Vol. 2, no. 3 , 6-27

»Von Reichenbach, María Cecilia y Aníbal Guillermo Bibiloni. 2012. Las dificultades de implantar una disciplina científica. Los primeros cincuenta años del Instituto de Física de La Plata. En Hurtado de Mendoza, Diego, ed. La física y los físicos argentinos: historias para el presente. Córdoba: Asociación Física Argentina; Universidad Nacional de Córdoba. p. 61-90. 


\section{O Fuentes}

» Biblioteca Central de la Universidad Nacional de La Plata. 1926. Clasificación sistemática del material bibliográfico: Instrucciones para el uso de los ficheros.

"Biblioteca Pública de la Universidad Nacional de La Plata. s.f. Guía para los trabajos de clasificación y catalogación bibliográfica en la biblioteca de la Universidad de la Plata.

»Comunicado interno de la Resolución del 27 de Enero de 1921. Biblioteca Pública de la Universidad Nacional de La Plata.

»Comunicado interno de la Resolución del 6 de Junio de 1921. Biblioteca Pública de la Universidad Nacional de La Plata.

» Resolución № 1875. Universidad Nacional de La Plata. 1919.

» Simons, Hanny. 1949. Al señor director de la Biblioteca Pública de la Universidad Nacional de La Plata. Copia en posesión del Centro de Documentación de la Inmigración de Habla Alemana en la Argentina (DIHA). 
